\title{
The Induction of Electric Currents in a Conducting Spherical Cap with Geomagnetic Applications: A Semi-analytical Approach for the Axisymmetric Case
}

\author{
A.A. Ashour, M.S. Abou-Dina and A.F. Ghaleb*
}

Department of Mathematics, Faculty of Science, Cairo University, Giza, Egypt, "Email: afghaleb@gmail.com.

ABSTRACT: The objective is to introduce a semi-analytical method for solving axisymmetric problems of electromagnetic induction in thin spherical caps placed in a time-varying magnetic field due to an axial magnetic dipole or in a time-varying uniform axial magnetic field. This method provides approximate solutions to mathematically difficult mixed boundary-value problems governing the induction of electric currents in thin sheets, for arbitrary angles of the cap. Numerical results are given and discussed. The best approximations were obtained for sheets with integrated conductivity decreasing to zero towards the edge of the sheet. The case of uniform conductivity, characterized by weak singularities of the induced magnetic vector potential at the rim of the cap, yielded relatively large errors and is dealt with separately within an improved model. The method may be adequately extended to deal with other problems involving more complicated geometries, arbitrary electric conductivity distributions and inducing magnetic fields, in two or three dimensions, for various geophysical applications.

KEYWORDS: Spherical cap, Geomagnetic applications, Axisymmetric case.

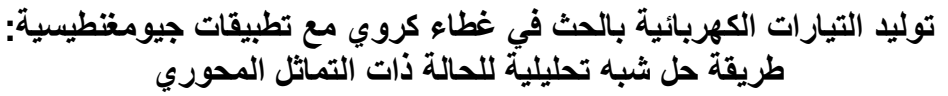

عطية عبدالسلام عاشور، مصطفى صابر أبو دينة وأحمد فؤاد غالب

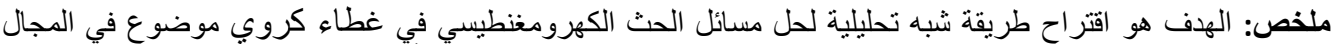

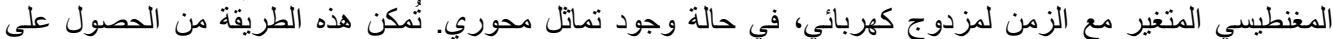
حلول تقريبية لمسائل الثروط الحدية المعقدة التي تصف نوليد فئي التيارات الكهربائية في الرقائق الموصلة للكهرباء التي تكون

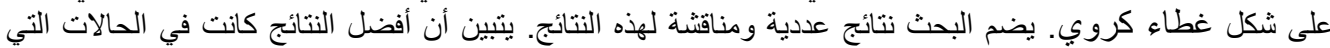

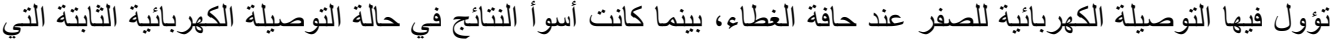

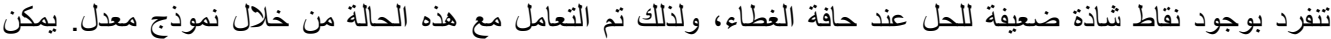

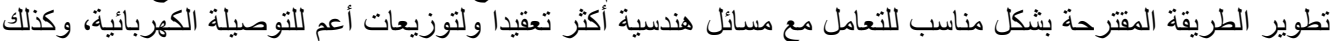
لأنو اع متعددة من المجالات الحاثة، في بعدين أو ثلاثة لتطبيقات جيوفيزيائية مختلفة.
} 


\section{INDUCTION OF ELECTRIC CURRENTS IN A CONDUCTING SPHERICAL CAP}

\section{Introduction}

$\mathbf{M}^{2}$

any of the geomagnetic phenomena of electromagnetic induction in Earth and in the surrounding Ionosphere are investigated through mathematical models involving the solution of difficult boundaryvalue problems in three-dimensional regions of different geometries. These formulations are considerably simplified by introducing the so-called "thin sheet" approximation, within which numerous applications can be considered. Early studies of induction of electric currents in uniformly conducting infinite, or finite plane sheets were undertaken within simplified models by Lamb, Maxwell and others.

The mathematical problem of electromagnetic induction in thin sheets was first formulated by Price (1949) in terms of the scalar magnetic potentials of the inducing and the induced magnetic fields. A method suggested by Price was realized by Ashour (1950) to investigate the problem of induction of electric currents in a uniformly conducting circular disk. The method of multiform potentials was used by Ashour (1952) to resolve the induction problem in a uniform circular disk by the sudden creation of magnetic poles, which has application in the study of magnetic storms.

Price's formulation found numerous geophysical applications, such as the investigation of the variations of the magnetic currents induced in the oceans and in the Ionosphere. The sheet boundary condition derived by Price, however, involves the gradient along the sheet of the function representing the integrated conductivity. This renders the use of the condition most inconvenient when dealing with models involving discontinuities in the integrated conductivity, such as induction in uniform infinite strips and spherical or spheroidal caps, etc. It is such geometries, among others, which have direct geophysical application, for example when studying the coast line effect and simulating vast oceans, either as part of a spherical shell or of a plane sheet.

Motivated by this limitation in Price's formulation, Ashour (1965) proposed a sheet boundary condition in terms of the vector potential, in which only the conductivity of the sheet appears, but not its gradient. This formula is specially useful for axisymmetric and for two-dimensional problems, whether the conductivity of the sheet is continuous or not, as the vector potential has only one non-vanishing component involving two of the coordinates.

An early attempt to resolve the induction problem while avoiding dual integral or dual series equations was undertaken by Ashour (1950). The same author, Ashour (1971), deduces an integral equation for the current density induced in a thin, uniformly conducting circular disk or in an infinite plane sheet with a circular hole, and obtains solutions for the infinitely conducting sheet. The Fredholm integral equation giving the density of the induced currents in a thin spherical cap was also deduced in (Ashour, 1971) from the case of the disc and a solution was found for the infinitely conducting cap for certain special conductivity distributions. Doss and Ashour (1971) investigated the electric currents induced in a hemispherical cap by axisymmetric time-varying fields and discussed the case of highly conducting caps. The case of two thin half-sheets was considered by Weidelt (1971) who succeeded in unveiling the logarithmic singular behaviour of the normal component of the magnetic field at the line of separation for the considered two-dimensional configuration. Hutson et al. (1972) obtained an integral equation for the density of the induced currents in sheets of arbitrary shapes and for a wide class of conductivity distributions. They discussed the existence and uniqueness of the solution and the convergence of the proposed iteration method. They also noticed the necessity for introducing a scalar electric potential together with the vector potential, in accordance with the general rules of electrodynamics. These authors produced numerical results for the induced currents in a hemisphere of constant electric conductivity and compared their results with an exact solution. However, they did not show results at the immediate vicinity of the rim, neither have they produced results for the magnetic field components. Ashour (1974a) further investigated this scalar electric potential and showed that it reduces to a constant for axisymmetric and for twodimensional problems. He deduced a generalized boundary condition on the sheet. Exact solutions for certain axisymmetric problems of induction in thin non-uniform sheets with special distributions of conductivity were given by Ashour (1974b) using inversion. Three-dimensional modelling and inverse problems were discussed by Hewson-Browne and Kendall (1976). These authors pointed out the importance of developing theoretical investigations in order to produce new methods of solution for the induction problems. Fainberg (1980) gave an 


\section{A.A. ASHOUR, M.S. ABOU-DINA and A.F. GHALEB}

ample survey of the state of global investigations of electromagnetic induction in geophysics.

For those problems where the sheet is uniform and forms a part of a closed coordinate surface (spherical cap, oblate or prolate spheroidal cap, disc, infinite strip, half-plane, etc), the mathematical problem reduces to finding the solution of dual (triple, etc) integral equations or series equations. The analytical solution in closed form of such equations is not usually available and runs into serious mathematical difficulties, especially in those cases where the conductivity function has a discontinuity at the edge of the sheet. The anomalous behaviour of the normal magnetic field was discussed by many authors. Ashour (1965) and later on Raval et al.(1981) noticed that the normal component of the magnetic field at the egde of the perfect conductor behaves like $r^{-1 / 2}$, where $r$ denotes the distance from the edge. As stated above, Weidelt (1971) uncovered the logarithmic singularity at the edge of two half-plane conductors. Wolf $(1983 \mathrm{a}, 1983 \mathrm{~b})$ discussed this issue. He notes that the model of a perfect conductor is poor at low frequencies and induction ceases to be important, hence the limitation of this model for geophysical applications.

Rigorous solutions to induction problems in thin sheets of finite electric conductivity could be obtained only in few cases of axisymmetric and two-dimensional problems (see, for example (Ashour, 1971), (Doss and Ashour, 1971), (Hewson-Browne and Kendall, 1976) and (Hutson et al., 1972)). The significance of configurations involving perfect conductors as useful idealizations of real conductors was underlined in (Hewson-Browne and Kendall, 1976).

The difficulty of the mathematical problem and the constant need for more elaborate mathematical models to deal with modern geophysical observations boosted research towards iterative techniques and purely numerical methods, in particular for 3D modelling. Review articles may be found in (Chave and Booker, 1987; Gainberg et al., 1990; Tarits, 1994; Varentsov, 1983). Siew and Hurley (1988) treated the problem of the disc under a non-axisymmetric inducing field by an orthogonal expansion method. For applications of spherical cap harmonic analysis, one may refer to (Thebault et al., 2004, 2006a, 2006b).

The aim of the present work is to fill the existing gap between analytical and numerical approaches by presenting an efficient, semi-analytical procedure capable of handling models of arbitray angle of the cap and for a wide class of the conductivity function and the inducing magnetic field. The method can be adequately extended to cover other geometries of the sheet. The essence of the method is to use a boundary expansion for a function representing the error between the actual boundary conditions and those resulting from the proposed formula for the approximate solution. The method is a variant of Trefftz's collocation method and was earlier initiated and implemented for efficiency in a series of test problems by two of the authors (Abou-Dina and Ghaleb, 2004). In the present case, this is used in combination with the usual collocation technique.

The problem under consideration is that of a thin, spherical cap of electric (integrated) conductivity, placed in the time-varying magnetic field due to an axisymmetric magnetic dipole. The case of a time-varying axial uniform magnetic field is also considered. Other types of axisymmetric inducing fields may be treated within the same formulation. Our treatment, however, will be confined to those cases of practical interest, when the effects of induction are appreciable in size. The solution of the considered problem may be used to evaluate the currents induced in the Earth by the change of the ionospheric currents, as well as the components of the induced magnetic field. This model was previously investigated by Doss and Ashour (1971), who reduced the problem of a hemispherical shell to that of a non-uniform circular disc, then resolved the resulting dual integral equations by successive approximations, only for high values of a non-dimensional physical parameter characterizing the problem, corresponding to high conductivity or/and to very rapid variations of the inducing magnetic field. In our formulation, the conductivity takes on finite values, not necessarily high, and the time variations of the inducing magnetic field may also be taken to be arbitrary. The angle of the cap can also vary arbitrarily, but not be larger than $90^{\circ}$. The case of uniform conductivity is treated separately. Thus, the solutions obtained from the present formulation are expected to provide a better description of the geophysical phenomenon.

\section{Statement of the problem}

Consider a thin, spherical cap defined by $r=a$ and $0 \leq \theta \leq \alpha$ with respect to a system of spherical polar 


\section{INDUCTION OF ELECTRIC CURRENTS IN A CONDUCTING SPHERICAL CAP}

coordinates $(r, \theta, \varphi)$ with the elevation angle $\theta$ measured from the upward vertical. The integrated electric conductivity $\sigma(\theta)$ of the cap is an arbitrary continuous function of the angle $\theta$. The cap is surrounded by a dielectric medium. A time varying magnetic dipole of moment $M(t) \mathbf{k}$ is situated at a distance $c$ from the origin, on the negative part of the $z$-axis $(\theta=\pi)$. Here $t$ denotes the time. Accordingly, a system of electric currents is induced in the cap and it is required to calculate the intensity of the resulting current in the cap and the magnetic field everywhere in space. The case of an inducing, time varying, uniform axial magnetic field is also treated in the numerical examples.

\section{Basic equations and boundary conditions}

The problem of electromagnetic induction in thin sheets was initiated by Price (1949) using a formulation through the magnetic scalar potential function. The sheet boundary condition derived within this formulation involves the derivatives of the electric conductivity of the sheet. This makes the procedure incapable of dealing with models involving discontinuities in the conductivity. A remedy to this situation was proposed by Ashour (1965), who replaces the magnetic scalar potential by the magnetic vector potential, thus making the new sheet boundary condition derivative-free. Ashour's formulation could handle two-dimensional problems, as well as problems with axial symmetry. A generalization of the sheet boundary condition was later suggested by Ashour (1974) to cover the general case. Many applications may be found in the literature (Ashour, 1974).

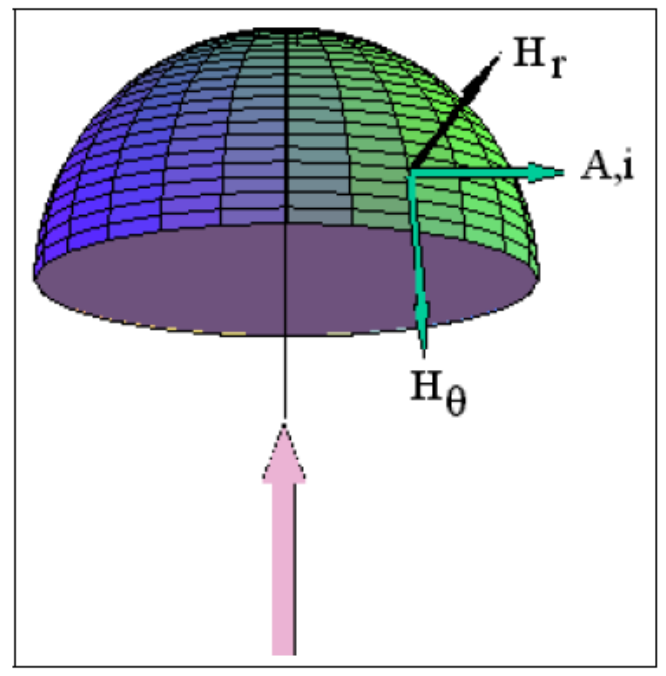

Figure 1. Geometry of the problem.

The basic field equations to be used are those of classical electrodynamics in the quasi-static approximation, expressed through the magnetic vector potential (no scalar magnetic potential is needed here due to axial symmetry), together with the usual boundary conditions. These relations may be found in standard textbooks (Jackson, 1962). In what follows, we use the rationalized MKS system of units and the magnetic permeability in the dielectric is taken as equal to unity.

Let us assume that the function of electrical conductivity has the form

$$
\sigma=\sigma_{0} \zeta(u), \quad u=\cos \theta
$$




\section{A.A. ASHOUR, M.S. ABOU-DINA and A.F. GHALEB}

in the used system of spherical polar coordinates $(r, \theta, \varphi)$ in which the sheet is part of a coordinate surface. For these special choices of the conductivity and the inducing magnetic fields, the problem under consideration presents axial symmetry about the axis of the cap. In such cases, it is well-known (Doss and Ashour, 1971) that the vector potential $\mathbf{A}$ for any of the inducing and the induced magnetic fields, as well as the electric current in the cap, have only one non-vanishing component in the direction of $\varphi$-increasing as shown on Figure 1 . These components are independent of the angle $\varphi$. Hence

$$
\mathbf{A}=A(r, \theta) \mathbf{e}_{\varphi},
$$

where $\mathbf{e}_{\varphi}$ is the unit vector in the direction of $\varphi$-increasing. Hereafter, the superscripts $(e)$ and $(i)$ refer to quantities belonging to the inducing and to the induced fields respectively.

For the considered inducing fields, an axial dipole or an axial uniform field, it is well-known that the function $A^{(e)}(r, \theta)$ is given by Wolf (1983b, p.143)

$$
A^{(e)}(r, \theta)=\frac{\mu_{0} M(t)}{4 \pi} \frac{r \sin \theta}{\left(r^{2}+2 c r \cos \theta+c^{2}\right)^{3 / 2}}, \quad M(t)=M_{0} e^{i p t}
$$

for the magnetic dipole of moment $M(t)$ with magnetic field components

$$
\begin{aligned}
& H_{r}^{(e)}(r, \theta)=\frac{M(t)}{4 \pi} \frac{2\left(r^{2}+2 c r \cos \theta+c^{2}\right)+3 c r \sin ^{2} \theta}{\left(r^{2}+2 c r \cos \theta+c^{2}\right)^{5 / 2}}, \\
& H_{\theta}^{(e)}(r, \theta)=\frac{M(t) \sin \theta}{4 \pi} \frac{r^{2}-c r \cos \theta-2 c^{2}}{\left(r^{2}+2 c r \cos \theta+c^{2}\right)^{5 / 2}},
\end{aligned}
$$

and

$$
A^{(e)}(r, \theta)=\frac{\mu_{0} H_{0}(t)}{2} r \sin \theta,
$$

for the axial uniform field of intensity $H_{0}(t)$, with magnetic field components

$$
\begin{aligned}
& H_{r}^{(e)}(r, \theta)=H_{0}(t) \cos \theta, \\
& H_{\theta}^{(e)}(r, \theta)=-H_{0}(t) \sin \theta
\end{aligned}
$$

In both cases, the magnetic field component in the direction of $\varphi$-increasing vanishes. The directions of the other two non-vanishing components are shown on Figure 1.

As for the vector potential of the induced magnetic field, it must be everywhere finite and continuous, and satisfies the vectorial Laplace's equation

$$
\nabla^{2} \mathbf{A}^{(i)}=\mathbf{0}
$$

From the above equations and conditions, and using the well-known representations of harmonic functions in the system of spherical polar coordinates, one may write down the solution for the case of the axial magnetic dipole (Doss and Ashour, 1971)

$$
A^{(i)}(r, \theta)=\frac{\mu_{0} M(t)}{4 \pi a^{2}} \begin{cases}\sum_{n=1}^{\infty} a_{n}\left(\frac{r}{a}\right)^{n} P_{n}^{1}(u), & r \leq a, \\ \sum_{n=1}^{\infty} a_{n}\left(\frac{r}{a}\right)^{-n-1} P_{n}^{1}(u), & r>a,\end{cases}
$$




\section{INDUCTION OF ELECTRIC CURRENTS IN A CONDUCTING SPHERICAL CAP}

where $a_{n}, n=1,2, \cdots$ are coefficients to be determined, $P_{n}^{1}$ is the associated Legendre function of degree $n$ and order 1 . For the uniform inducing field, $M(t)$ is replaced by $4 \pi a^{3} H_{0}(t)$.

It is known that

$$
P_{n}^{1}(u)=\left(1-u^{2}\right)^{1 / 2} \frac{d P_{n}(u)}{d u}
$$

and $P_{n}(u)$ is the Legendre polynomial of degree $n$ given by Rodrigues' formula

$$
P_{n}(u)=\frac{1}{2^{n} n !} \frac{d^{n}}{d u^{n}}\left(u^{2}-1\right)^{n}
$$

The sheet boundary condition as given by Ashour (1965) reduces in the present case to:

$$
\left(\frac{\partial A^{(i)}}{\partial r}\right)_{+}-\left(\frac{\partial A^{(i)}}{\partial r}\right)_{-}=\mu_{0} \sigma_{0} \zeta D\left(A^{(e)}+A^{(i)}\right), \quad r=a, \quad 0 \leq \theta \leq \alpha
$$

where $(+)$ and $(-)$ signs refer to the quantities calculated on the sheet for $r=a+0$ and $r=a-0$ respectively, $D$ denotes the operator of differentiation with respect to the time $t$ and $\mu_{0}$ is the magnetic permeability of free space with value

$$
\mu_{0}=4 \pi \times 10^{-7} \mathrm{Hm}^{-1}
$$

On the remaining part of the sphere, i.e. for $r=a, \alpha<\theta \leq \pi$, the normal derivative $\partial A^{(i)} / \partial r$ is continuous, which is equivalent to the no current condition.

From equations (5) and (6) and the continuity of the magnetic field outside the sheet, the coefficients $a_{n}, n=1,2, \cdots$ are found to satisfy the dual series equations:

$$
\begin{aligned}
\sum_{n=1}^{\infty}[ & \left.\frac{2 n+1}{\Lambda}+\zeta(u)\right] a_{n} P_{n}^{1}(u)=-\zeta(u) A^{(e)}(a, u), \quad \cos \alpha \leq u \leq 1, \\
& \sum_{n=1}^{\infty}(2 n+1) a_{n} P_{n}^{1}(u)=0, \quad-1 \leq u<\cos \alpha,
\end{aligned}
$$

where

$$
\Lambda=\mu_{0} \sigma_{0} a D
$$

The term involving $\Lambda$ in the denominator in (7a) may be interpreted, as usual, as an inverse operator and evaluated according to the rules of operational calculus. Without loss of generality, the dependence of the inducing field on time may be taken as a multiplicative term $e^{i \omega t}$ with constant $\omega$. Thus, the inducing field has a periodic time variation of frequency $v=\omega / 2 \pi$, in which case the operator $\Lambda$ acting on a function of time will be replaced by a multiplicative, dimensionless factor

$$
\Lambda_{0}=i \mu_{0} \sigma_{0} a \omega
$$

An advantage of the above formulation of the sheet boundary condition is that the case of a perfect conductor is obtained as a limiting case when $\Lambda \rightarrow \infty$.

The only non-vanishing components of the induced magnetic field are given in terms of the coefficients $a_{n}, n=1,2, \cdots$ by the expressions

$$
H_{r}^{(i)}(r, \theta)=\frac{1}{\mu_{0}} \frac{1}{r}\left(\frac{\partial A^{(i)}}{\partial \theta}+\cot \theta A^{(i)}\right)
$$




$$
\begin{gathered}
=\left\{\begin{array}{l}
-\frac{1}{4 \pi a^{3}} M(t) \sum_{n=1}^{\infty} n(n+1) a_{n}\left(\frac{r}{a}\right)^{n} P_{n}(u), r<a, \\
-\frac{1}{4 \pi a^{3}} M(t) \sum_{n=1}^{\infty} n(n+1) a_{n}\left(\frac{r}{a}\right)^{-n-2} P_{n}(u), r>a
\end{array}\right. \\
H_{\theta}^{(i)}(r, \theta)=-\frac{1}{\mu_{0}}\left(\frac{\partial A^{(i)}}{\partial r}+\frac{1}{r} A^{(i)}\right) \\
=\left\{\begin{array}{l}
\frac{1}{4 \pi a^{3}} M(t) \sum_{n=1}^{\infty}(n+1) a_{n}\left(\frac{r}{a}\right)^{n-1} P_{n}^{1}(u), r \leq a, \\
\frac{1}{4 \pi a^{3}} M(t) \sum_{n=1}^{\infty} n a_{n}\left(\frac{r}{a}\right)^{-n-2} P_{n}^{1}(u), r>a
\end{array}\right.
\end{gathered}
$$

The current density in the sheet is found from Ampère's circuital relation as

$$
\begin{aligned}
i_{\varphi}(\theta)=\left(H_{\theta}^{(i)}\right)_{+} & -\left(H_{\theta}^{(i)}\right)_{-}=-\frac{1}{\mu_{0}}\left[\left(\frac{\partial A^{(i)}}{\partial r}\right)_{+}-\left(\frac{\partial A^{(i)}}{\partial r}\right)_{-}\right] \\
& =\frac{M(t)}{4 \pi a^{3}} \sum_{n=1}^{\infty}(2 n+1) a_{n} P_{n}^{1}(\cos \theta), \quad 0 \leq \theta \leq \alpha .
\end{aligned}
$$

The dual series equations $(7 \mathrm{a}, \mathrm{b})$ were previously solved in certain particular cases, usually by transforming them through lengthy manipulations to a single Fredholm integral equation for an auxiliary function which, in turn, is solved either analytically in a very restraint number of cases (Fainberg, 1980) or by further reduction to an infinite system of linear algebraic equations solved numerically. An alternative method is proposed here below. This is a variant of a more general method developed earlier by two of the authors (M.S. Abou-Dina and A.F. Ghaleb), relying on a boundary Fourier expansion (Wolf, 1983a).

\section{The method}

Looking for an approximate solution to the dual series equations (7), we start by considering the truncated dual series equations

$$
\begin{gathered}
\sum_{n=1}^{N}\left[\frac{2 n+1}{\Lambda}+\zeta\right] a_{n}^{(N)} P_{n}^{1}(u)+\zeta A^{(e)}(a, u)=0, \quad \cos \alpha \leq u \leq 1, \\
\sum_{n=1}^{N}(2 n+1) a_{n}^{(N)} P_{n}^{1}(u)=0, \quad-1 \leq u<\cos \alpha,
\end{gathered}
$$

where $a_{n}^{(N)}, n=1,2, \cdots, N$ are the unknowns and $N$ is a predetermined, sufficiently large positive integer. As $N \rightarrow \infty$, system (11) tends to system(7) and $a_{n}^{(N)} \rightarrow a_{n}$ for all $n=1,2, \cdots$.

Earlier work on two-dimensional problems or three-dimensional axisymmetric problems of electromagnetic induction in finite thin sheets of uniform conductivity (Doss and Ashour, 1971; Weidelt, 1971) indicates a singular behaviour of the induced magnetic field at the boundary of the sheet. The component $H_{r}^{(i)}$ of the magnetic field has a singularity when crossing the cap along the $\theta$-direction (the corresponding singularity for plane problems is of logarithmic nature at the edge of discontinuity of the electrical conductivity, 


\section{INDUCTION OF ELECTRIC CURRENTS IN A CONDUCTING SPHERICAL CAP}

cf (Doss and Ashour, 1971), while the component $H_{\theta}^{(i)}$ has a jump there. The method presented here below cannot deal efficiently with such a situation, which will be treated separately in a later section. Thus, for the moment, it is assumed that the function defining the conductivity is bounded and continuous on the sheet and that it decreases to zero towards the edge of the cap.

Let us denote the functions in the left-hand sides of equations (11) by $F^{(N)}\left(u_{1}\right)$ and $G^{(N)}\left(u_{2}\right)$ respectively, with $\cos \alpha \leq u_{1} \leq 1$ and $-1 \leq u_{2} \leq \cos \alpha$. These two functions are expanded in their domains of definition in terms of two orthogonal families of functions $P_{r}^{1}[w]$ and $P_{s}^{1}[z]$ respectively, $r=0,1,2, \cdots$ and $s=1,2, \cdots$, as:

$$
F^{(N)}\left[u_{1}(w)\right]=\sum_{m_{1}=1}^{\infty} r_{m_{1}}^{(N)} P_{m_{1}}^{1}[w], \quad-1 \leq w \leq 1,
$$

and

$$
G^{(N)}\left[u_{2}(z)\right]=\sum_{n=1}^{\infty} s_{m_{2}}^{(N)} P_{m_{2}}^{1}[z], \quad-1 \leq z \leq 1
$$

with

$$
u_{1}=1+\frac{1-\cos \alpha}{2}(w-1), \quad u_{2}=-1+\frac{1+\cos \alpha}{2}(z+1) .
$$

One has

$$
r_{m_{1}}^{(N)}=\frac{2 m_{1}+1}{2 m_{1}\left(m_{1}+1\right)} \int_{-1}^{1} F^{(N)}\left[u_{1}(w)\right] P_{m_{1}}^{1}(w) d w, \quad m_{1}=1,2, \cdots
$$

and

$$
s_{m_{2}}^{(N)}=\frac{2 m_{2}+1}{2 m_{2}\left(m_{2}+1\right)} \int_{-1}^{1} G^{(N)}\left[u_{2}(z)\right] P_{m_{2}}^{1}(z) d z, \quad m_{2}=1,2, \cdots
$$

As $N \rightarrow \infty$ (for the exact solution), the coefficients $r_{m_{1}}^{(N)}$ and $s_{m_{2}}^{(N)}$ tend to zero for all values of $m_{1}$ and $m_{2}$. However, for an approximate solution, one needs only set to zero a finite number of these coefficients, i.e. one sets

$$
\int_{-1}^{1} F^{(N)}\left[u_{1}(w)\right] P_{m_{1}}^{1}(w) d w=0, \quad m_{1}=1,2, \cdots, M_{1}
$$

and

$$
\int_{-1}^{1} G^{(N)}\left[u_{2}(z)\right] P_{m_{2}}^{1}(z) d z=0, \quad m_{2}=1,2, \cdots, M_{2}
$$

for some prefixed $M_{1}$ and $M_{2}$. Let

$$
M=M_{1}+M_{2}
$$

Insertion of expressions (12) for $F^{(N)}\left[u_{1}(w)\right]$ and $G^{(N)}\left[u_{2}(z)\right]$ into conditions (14) yields the following system of linear, algebraic equations in the unknowns $a_{n}^{(N)}, n=1,2, \cdots, N$, written in matricial form as

$$
A \mathbf{X}=\mathbf{B},
$$

where $\mathbf{X}$ is the vector formed by the unknown coefficients $a_{n}^{(N)}, A$ is an $(M \times N)$ matrix, $\mathbf{B}$ is an $(M \times 1)$ vector defined blockwise by 


\section{A.A. ASHOUR, M.S. ABOU-DINA and A.F. GHALEB}

$$
A=\left[\begin{array}{l}
A^{(1)} \\
A^{(2)}
\end{array}\right], \quad B=\left[\begin{array}{l}
B^{(1)} \\
B^{(2)}
\end{array}\right],
$$

where $A^{(1)}, A^{(2)}, B^{(1)}$ and $B^{(2)}$ have respective dimensions $\left(M_{1} \times N\right),\left(M_{2} \times N\right),\left(M_{1} \times 1\right)$ and $\left(M_{2} \times 1\right)$, and

$$
\begin{gathered}
A_{m_{1} n}^{(1)}=\frac{2 n+1}{\Lambda} \int_{-1}^{1} P_{n}^{1}\left[u_{1}(w)\right] P_{m_{1}}^{1}(w) d w \\
+\int_{-1}^{1} \zeta\left[u_{1}(w)\right] P_{n}^{1}\left[u_{1}(w)\right] P_{m_{1}}^{1}(w) d w, \quad m_{1}=1,2, \cdots, M_{1} \\
A_{m_{2} n}^{(2)}=(2 n+1) \int_{-1}^{1} P_{n}^{1}\left[u_{2}(z)\right] P_{m_{2}}^{1}(z) d z, \quad m_{2}=1,2, \cdots, M_{2}, \\
B_{m_{1}}^{(1)}=-\int_{-1}^{1} \zeta\left[u_{1}(w)\right] A^{(e)}\left[a, u_{1}(w)\right] P_{m_{1}}^{1}(w) d w, \quad m_{1}=1,2, \cdots, M_{1},
\end{gathered}
$$

and

$$
B_{m_{2}}^{(1)}=0, \quad m_{2}=1,2, \cdots, M_{2}
$$

For finite $N, M_{1}, M_{2}$, the resulting values of the unknown coefficients are denoted $a_{n}^{\left(N, M_{1}, M_{2}\right)}$. The functions $F$ and $G$ defined above are correspondingly denoted $F^{\left(N, M_{1}, M_{2}\right)}$ and $G^{\left(N, M_{1}, M_{2}\right)}$.

\subsection{Accuracy criterion}

We introduce the quantities

$$
E 1^{\left(N, M_{1}, M_{2}\right)}=\sqrt{\int_{-1}^{1}\left|F^{\left(N, M_{1}, M_{2}\right)}\left[u_{1}(w)\right]\right|^{2}} d w
$$

and

$$
E 2^{\left(N, M_{1}, M_{2}\right)}=\sqrt{\int_{-1}^{1}\left|G^{\left(N, M_{1}, M_{2}\right)}\left[u_{2}(z)\right]\right|^{2}} d z
$$

Clearly, $E 1^{\left(N, M_{1}, M_{2}\right)}$ and $E 2^{\left(N, M_{1}, M_{2}\right)}$ measure the error in satisfying the boundary conditions of the problem. They are generally non-zero, but tend to zero as $N \rightarrow \infty$, hence they may be taken as criteria for the accuracy of the method.

The best results are not necessarily obtained with a square matrix $A$, i.e. when $M=N$. Therefore, we will assume that $M \geq N$. When $M>N$, the system (15) is overdetermined and the matricial equation (15a) is either solved by Gaussian elimination, or else reduced to a square system by matrix multiplication from the left by the transpose matrix $A^{T}$ :

$$
\left(A^{T} A\right) \mathbf{X}=A^{T} \mathbf{B}
$$

the solution of which is obtained by any adequate method for the required approximate values of the coefficients $a_{n}^{\left(N, M_{1}, M_{2}\right)}, n=1,2, \cdots, N$.

From the computationl point of view, $N$ cannot be increased without limit to improve the solution. Alternatively, the process to be followed is:

(i) $N$ is given an initial value, then $M_{1}$ and $M_{2}$ are varied until the best result is obtained according to a prefixed criterion.

(ii) The value of $N$ is then increased by 1 and the steps in (i) are repeated.

(iii) The process is terminated when the optimal values of $M_{1}, M_{2}$ and $N$ are reached, meaning that the 


\section{INDUCTION OF ELECTRIC CURRENTS IN A CONDUCTING SPHERICAL CAP}

next iterations do not add substantially to the results.

An accuracy criterion may be conveniently taken in the form:

$$
\max \left(E 1^{\left(N, M_{1}, M_{2}\right)}, E 2^{\left(N, M_{1}, M_{2}\right)}\right)<\varepsilon,
$$

$\varepsilon$ is chosen sufficiently small according to the needed accuracy.

\section{Numerical results for the case of conductivity decreasing to zero towards the edge}

As stated earlier, the method allows to consider a wide range of values for the geometrical and the physical parameters of the problem. For geophysical applications, however, one usually sets:

$$
a=6.48 \times 10^{6} \mathrm{~m}, \quad \sigma_{0}=2 \times 10^{4} \mathrm{~S}, \quad \omega=\frac{2 \pi}{24 \times 60 \times 60} \mathrm{~s}^{-1},
$$

corresponding to a spherical cap on the Earth's surface, an integrated conductivity of saline water in an ocean of depth $5 \mathrm{~km}$, and a 24-hour period for the inducing field.This gives the estimate

$$
|\Lambda| \simeq 12 \text {. }
$$

For definiteness, the function of electrical conductivity is taken in the form

$$
\zeta(u)=\left\{\begin{array}{cl}
\cos \left(\frac{\pi(u-1)}{2(\cos \alpha-1)}\right) & \text { if } u>\cos \alpha \\
0 & \text { otherwise }
\end{array}\right.
$$

and

$$
\alpha=\frac{\pi}{2}, \quad c=1 \quad \text { (for the dipole) }
$$

We have plotted the current density distribution $i(\theta)$, the induced magnetic vector potential and the magnetic field components $H_{r}^{(i)}(a+0, \theta), H_{\theta}^{(i)}(a, \theta)$ on the sphere $r=a$ using the optimal values of $M$ and $N$ when the inducing field is produced by an axial magnetic dipole or by an axial uniform magnetic field. The function of electrical conductivity is also shown on the same graph as $i(\theta)$. The computations were carried out for the values

$$
|\Lambda|=12,100
$$

The latter value corresponds to relatively high electrical conductivities and/or relatively rapid variations of the inducing magnetic field. For the best results:

$$
N=35, \quad M_{1}=M_{2} \sim 50
$$

We have noticed, however, that the results are improved if direct collocation is used on the part of the sphere defined by $r=a$ and $\alpha<\theta \leq \pi$. The optimal number of points placed on that interval was about 400 . The error in satisfying the dimensionless sheet boundary condition as given in (18) was of the order of 0.001 in all four cases shown below on Figures 2-5. 


\section{A.A. ASHOUR, M.S. ABOU-DINA and A.F. GHALEB}
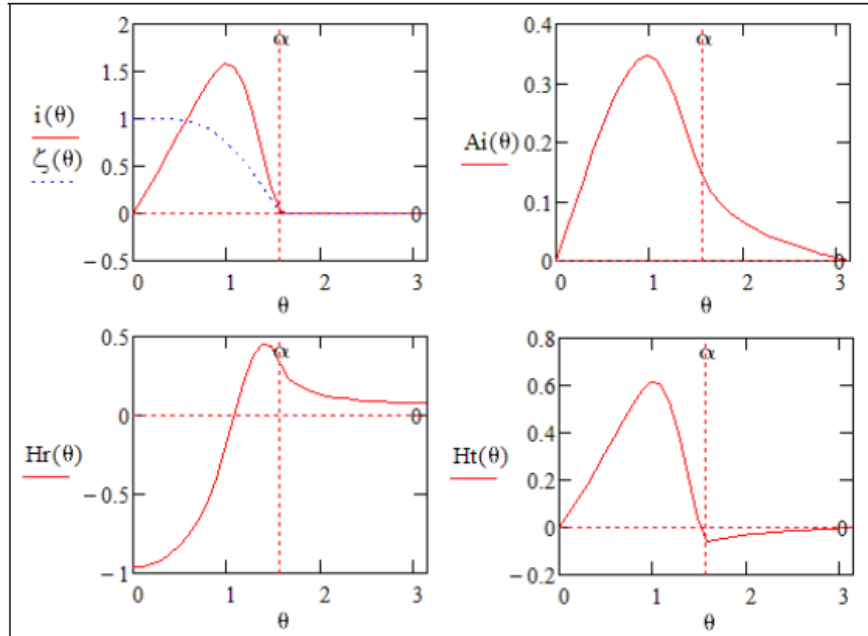

Figure 2. The results for conductivity decreasing to zero under a uniform axial magnetic field with $|\Lambda|=12$.

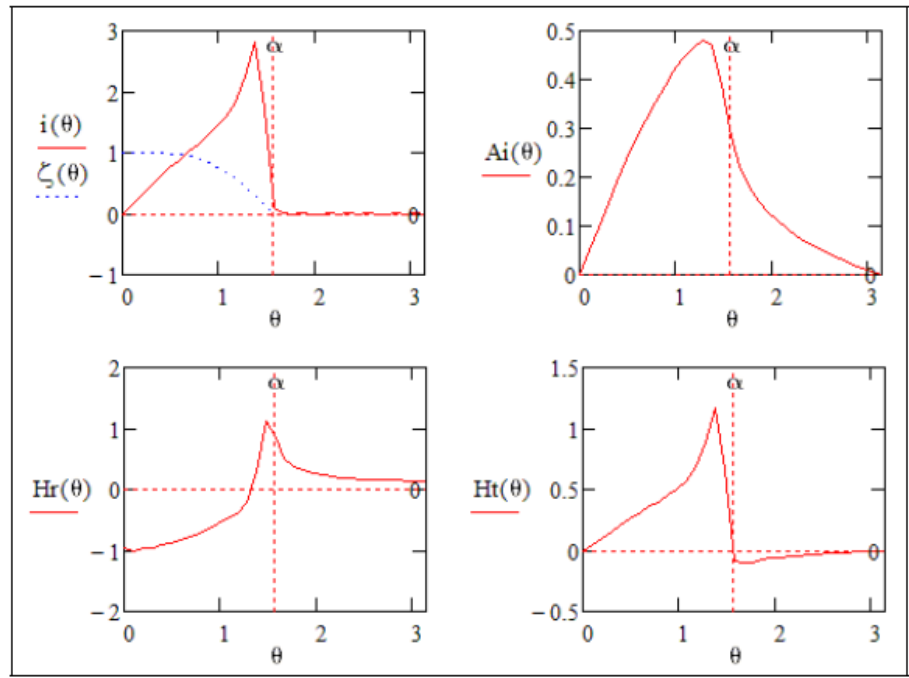

Figure 3. The results for conductivity decreasing to zero under a uniform axial magnetic field with $|\Lambda|=100$.

As expected, each of the functions $A^{(i)}(a, \theta), H_{\theta}^{(i)}(a, \theta)$ and $i(\theta)$ has one relative extremum on the sheet. The behavior of $i(\theta)$ and $H_{\theta}^{(i)}(a, \theta)$ is almost the same, except for the fact that the tangential component of the magnetic field is not required to vanish identically outside the sheet. It is worth noting and comparing the behavior of the normal component $H_{r}^{(i)}(a, \theta)$ around the rim of the cap in the two cases of the axial dipole and the axial magnetic field. An extrapolation to the case of uniform electrical conductivity seems to predict the following singular behavior in the vicinity of the rim: 


\section{INDUCTION OF ELECTRIC CURRENTS IN A CONDUCTING SPHERICAL CAP}

$$
H_{r}^{(i)}(a, \theta) \rightarrow+\infty \quad \text { when } \quad \theta \rightarrow \alpha \pm 0
$$

for the axial uniform magnetic field and

$$
H_{r}^{(i)}(a, \theta) \rightarrow \mp \infty \text { when } \theta \rightarrow \alpha \pm 0
$$

for the axial magnetic dipole. This result was confirmed by our calculations involving different functions of electrical conductivity with steeper descents towards the zero at the rim of the cap.

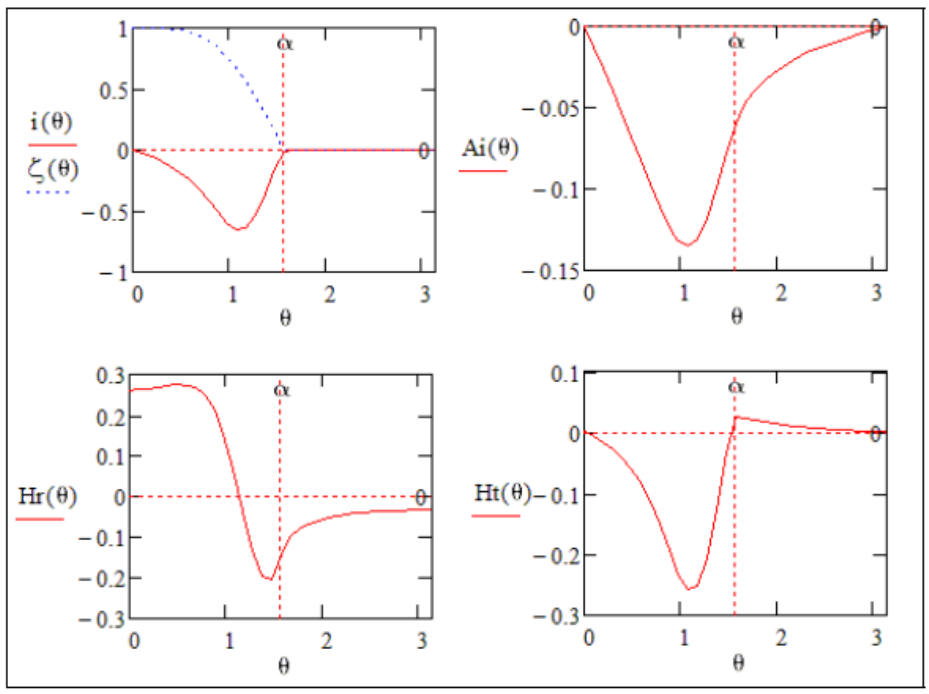

Figure 4. The results for conductivity decreasing to zero under a axial magnetic dipole with $|\Lambda|=12$.

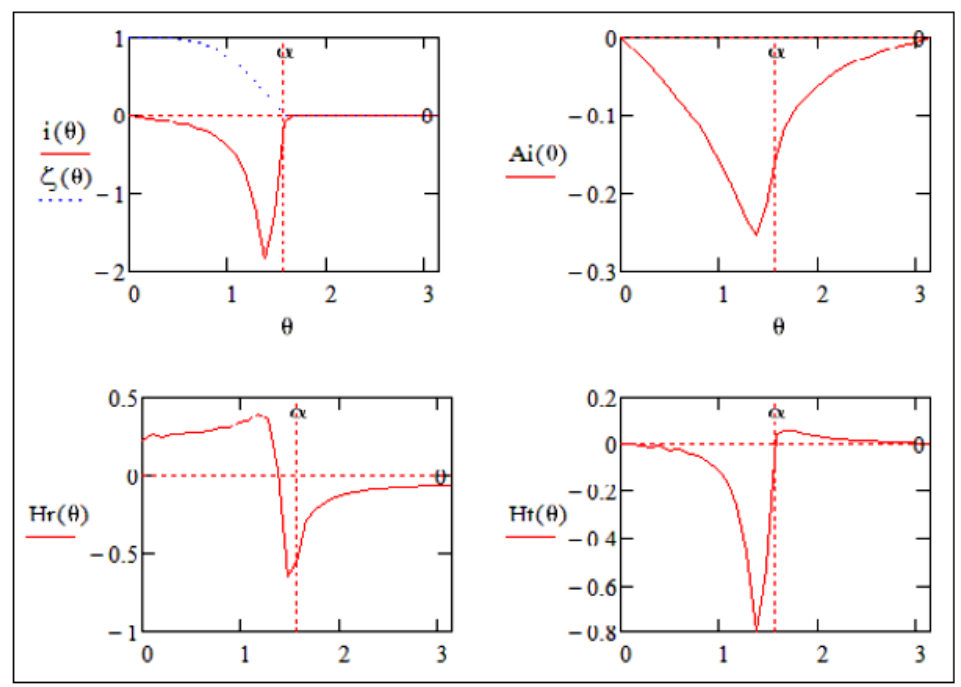

Figure 5. The results for conductivity decreasing to zero under a axial magnetic dipole with $|\Lambda|=100$. 


\section{A.A. ASHOUR, M.S. ABOU-DINA and A.F. GHALEB}

\section{An improved version for the uniform conductivity}

When the cap is of uniform electrical conductivity, the conductivity function is

$$
\zeta(u)=\left\{\begin{array}{lcr}
1 & \text { if } \quad u>\cos \alpha \\
0 & & \text { otherwise }
\end{array}\right.
$$

The method explained above does not yield good results. In order to simulate the singular behaviour of the magnetic field at the edge of the cap, we enrich the expansion (5a) with a solution of Laplace's equation on space, having a singularity at those points. Such a solution may be constructed as an expansion in the associated Legendre polynomials $P_{n}^{1}(\cos \theta)$ using the function $\operatorname{sgn}(\theta-\alpha)$. Other possibilities are currently under investigation. Thus,

$$
A^{(i)}(r, \theta)=M(t)\left\{\begin{array}{l}
\sum_{n=1}^{\infty} a_{n}\left(\frac{r}{a}\right)^{n} P_{n}^{1}(\cos \theta)+q g_{-}(r, \theta), \quad r \leq a, \\
\sum_{n=1}^{\infty} a_{n}\left(\frac{r}{a}\right)^{-n-1} P_{n}^{1}(\cos \theta)+q g_{+}(r, \theta), \quad r>a,
\end{array}\right.
$$

with

$$
\begin{gathered}
g_{-}(r, \theta)=\sum_{k=1}^{\infty} d_{k}\left(\frac{r}{a}\right)^{k} P_{k}^{1}(\cos \theta), \\
g_{+}(r, \theta)=\sum_{k=1}^{\infty} d_{k}\left(\frac{r}{a}\right)^{-k-1} P_{k}^{1}(\cos \theta)
\end{gathered}
$$

with properly chosen coefficients $d_{k}$ and $q$ is a multiplicative constant to be determined simultaneously with the coefficients $a_{n}$. In the new truncated dual series equations, the constant $q$ is identified with the coefficient $a_{N}^{(N)}$ and the dual series equations themselves take the form

$$
\begin{gathered}
\sum_{n=1}^{N-1}[(2 n+1)+\Lambda] a_{n}^{(N)} P_{n}^{1}(\cos \theta)+a_{N}^{(N)} G_{1}=-\Lambda A^{(e)}, \quad 0 \leq \theta \leq \alpha \\
\quad \sum_{n=1}^{N-1}(2 n+1) a_{n}^{(N)} P_{n}^{1}(\cos \theta)+a_{N}^{(N)} G_{2}=0, \quad \alpha<\theta \leq \pi,
\end{gathered}
$$

with

$$
\begin{gathered}
G_{1}(\theta)=\sum_{k=1}^{\infty} d_{k} P_{k}^{1}(\cos \theta)+\Lambda g_{ \pm}(a, \theta), \\
G_{2}(\theta)=G_{1}(\theta)-\Lambda g_{ \pm}(a, \theta)
\end{gathered}
$$




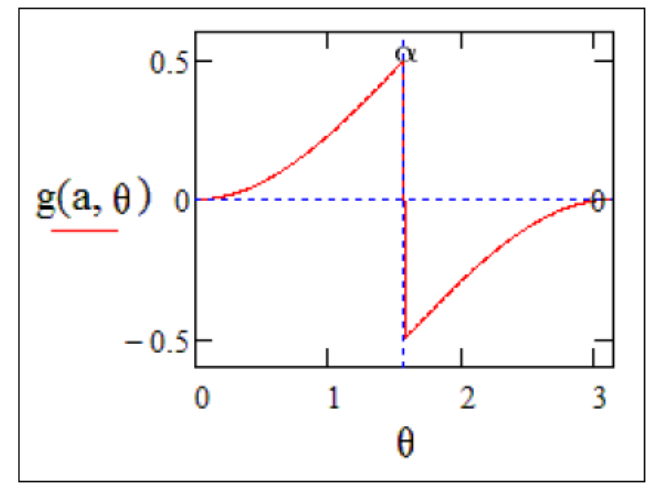

Figure 6. The singular solution on the sphere $r=a$.

Figure 6 shows the function $g(a, \theta)=g_{+}(a, \theta)=g_{-}(a, \theta)$.

\section{Numerical results for the case of uniform conductivity}

This improved version of the method was used to study the case of a hemisphere $\alpha=\pi / 2$ under a uniform, axial magnetic field previously investigated in (Chave and Booker, 1987) with $|\Lambda|=12$. The results of this improved version are shown on Figure 7.

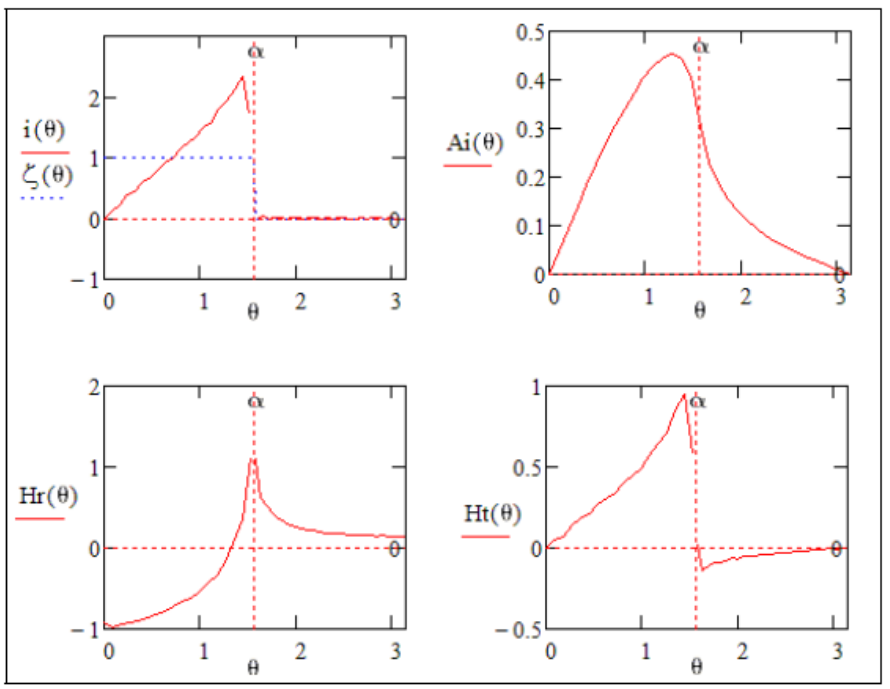

Figure 7. The results for a uniform conductivity under a uniform axial magnetic field with $|\Lambda|=12$.

Figure 8 compares our result for the real and imaginary parts of the electric current density, $\operatorname{Re}(i)$ and $\operatorname{Im}(i)$, with that of Hutson et al. (1972). The adjustment of the angle measure to degrees was made to match the reference. 


\section{A.A. ASHOUR, M.S. ABOU-DINA and A.F. GHALEB}

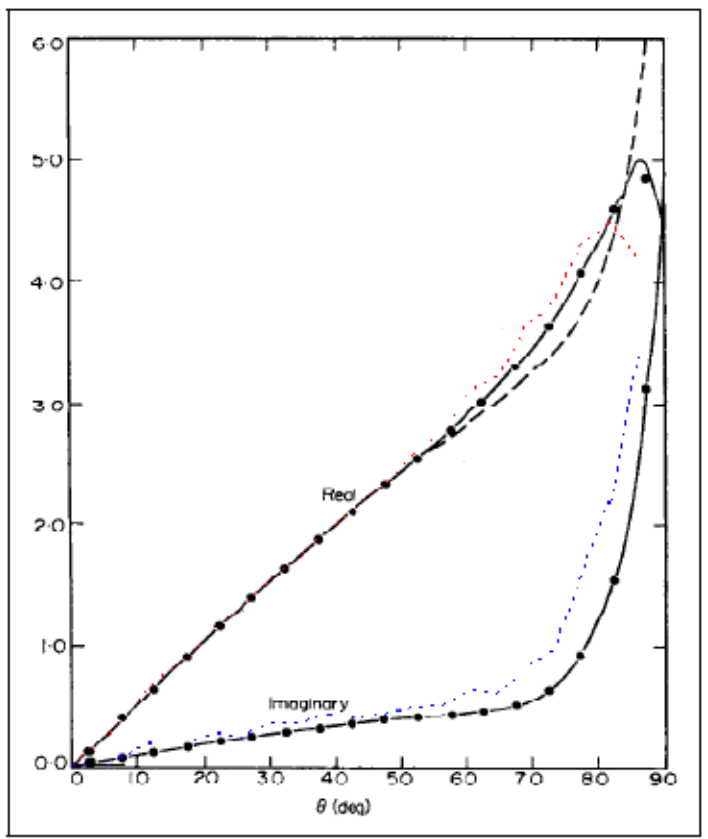

Figure 8. Comparison of present results with those of Chave and Booker (1987) for the real and imaginary parts of the current density.

The solid lines represent the exact solutions (this solution is not produced in (Hutson et al., 1972)) for the real and the imaginary parts of the electric current density, the dotted lines represent the numerical results of Hutson et al. (1972), while the dashed lines are those of the present work. The results obtained by the proposed method clearly deteriorate as one gets closer to the edge of the cap. As expected, the error in satisfying the sheet boundary condition is now of the order of 0.025 , i.e. much larger than for the case of the conductivity decreasing towards zero at the edge, and is concentrated towards the edge of the cap. Also, the number of points used for the collocation doubled in comparison to the previous case. We have considered the case of the axial magnetic dipole as well. The results are qualitatively similar to those of the uniform axial magnetic field, with a comparable order for the error.

\section{Conclusions}

The following conclusions were reached, concerning the efficiency of the present approach:

a. The proposed method, together with its improvement, can deal efficiently with the axisymmetric problem of magnetic induction in the spherical cap to generate approximate solutions to a difficult boundary-value problem for which very few exact solutions are known.

b. The method is flexible in what concerns the nature of the inducing magnetic field and the function of electrical conductivity in the cap, provided it is bounded and continuous.

c. There is room for increasing the accuracy by associating the usual collocation method with the one proposed above.

d. The error strictly decreases initially, but fails to keep this behavior after a threshold value of the number of degrees of freedom $N$ is reached: An oscillatory character generally establishes beyond this stage. This is probably due to the accumulation error. 


\section{INDUCTION OF ELECTRIC CURRENTS IN A CONDUCTING SPHERICAL CAP}

e. The method is more efficient for larger angles of the cap, as the induction effects become more pronounced. However, further studies are necessary when the cap exceeds the hemisphere.

f. The method becomes less efficient for larger $|\Lambda|$, i.e for higher electrical conductivity or/and faster time variations of the inducing magnetic field.

g. The level of accuracy is strongly affected by the presence of weak singularities located at the rim of the cap, in which case the expression for the approximate solution has to include additional terms accounting for these singularities.

h. Adequate harmonic functions with singularities at the edge could be introduced into the expansion set of functions in order to obtain improved approximate solutions. Such harmonic functions with boundary singularities may be efficiently used in other problems with more complicated geometries of the boundary.

i. The effect of the additionally introduced harmonic functions is to improve the accuracy and, simultaneously, to decrease significantly the number of terms in the expansion for the approximate solution and the number of zeroed Fourier coefficients.

j. The obtained results with geophysical applications yield results which are in qualitative agreement with the data scattered throughout the literature.

\section{References}

ABOU-DINA, M.S. and GHALEB, A.F. 2004. A Variant of Trefftz's Method by Boundary Fourier Expansion for Solving Regular and Singular Plane Boundary-Value Problems. J. Comp. Appl. Math. (C.A.M.), 167: 363-387.

ASHOUR, A.A. 1950. The Induction of Electric Currents in a Uniform Circular Disk. Quart. J. Mech. Appl. Math., 3(1): 119-128.

ASHOUR, A.A. 1952.The Induction of Electric Currents in a Uniformly Conducting Circular Disk by the Sudden Creation of Magnetic Poles. Quart. J. Mech. Appl. Math., 5(3): 379-384.

ASHOUR, A.A. 1965. Electromagnetic Induction in Finite Thin Sheets. Quart. J. Mech. Appl. Math., 18(1): $73-$ 86.

ASHOUR, A.A. 1971. Electromagnetic Induction in Thin Finite Sheets Having Conductivity Decreasing to Zero at the Edge, with Geomagnetic Applications. Geophys. J. R. Astr. Soc., 22: 489-498.

ASHOUR, A.A. 1974a. Research Note: The Condition at the Surface of a Thin Conductor in Which Currents are Induced. Geophys. J. R. Astr. Soc., 36: 235-237.

ASHOUR, A.A. 1974b. Exact Solutions for Certain Axisymmetric Problems of Induction in Thin Non-Uniform sheets. J. Geophys. Res., 79(16): 2479-2486.

CHAVE, A.D. and BOOKER, J.R. 1987. Electromagnetic Induction Studies. Reviews of Geophysics, 25(5): 9891003.

DOSS, S.S. and ASHOUR, A.A. 1971. Some Results on the Magnetic Field of Electric Currents Induced in a Thin Hemispherical Shell of Finite Conductivity, with Geomagnetic Applications. Geophys. J. R. Astr. Soc., 22: 385-400.

FAINBERG, E.B. 1980. Electromagnetic Induction in the World Ocean. Geophysial Surveys, 4: 157-171.

GAINBERG, E.B., KUVSHINOV, A.V. and SINGER, B.Sh. 1990. Electromagnetic Induction in a Spherical Earth with Non-uniform Oceans and Continents in Electric Contact with the Underlying Medium. Geophys. J. Int., 102: 273-281.

HEWSON-BROWNE, R.C. and KENDALL, P.C. 1976. Magnetotelluric Modelling and Inversion in Three Dimensions. Acta Geodet., Geophys. et Montanist. Acad. Sci. Hung., 11(3-4), 427-446.

HUTSON, V.C.L., KENDALL, P.C. and MALIN, S.R.C. 1972. Computation of the Solution of Geomagnetic Induction Problems: A General Method, with Applications. Geophys. J. R. Astr. Soc., 28: 417-443.

JACKSON, J.D. 1962. Classical Electrodynamics. John Wiley \& Sons, Inc., New York, London.

PRICE, A.T. 1949. The Induction of Electric Currents in Non-uniform Thin Sheets and Shells. Quart. J. Mech. 


\section{A.A. ASHOUR, M.S. ABOU-DINA and A.F. GHALEB}

Appl. Math., 2(3): 283-310.

RAVAL, U., WEAVER, J.T. and DAWSON, T.W. 1981. The Ocean Coast-effect Re-examined. Geophys. J. R. Astr. Soc., 67: 115-123.

SIEW, P.F. and HURLEY, D.G. 1988. Electromagnetic Response of Thin Disks. J. Appl. Math. and Phys. (ZAMP), 39: 619-633.

TARITS, P. 1994. Electromagnetic Studies of Global Geodynamic Processes. Surveys in Geophysics, 15: 20238.

THEBAULT, E., SCHOTT, J.J., MANDEA, M. and HOFFBECK, J.P. 2004. A New Proposal for Spherical Cap Harmonic Modeling. Geophys. J. Int., 159: 83-103.

THEBAUlT, E., SCHOTT, J.J. and MANDEA, M. 2006a. Revised Spherical Cap Harmonic Analysis: Validation and Properties. J. Geophys. Res.-Solid Earth, 111(5): B01102, 1-17.

THEBAULT, E., MANDEA, M. and SCHOTT, J.J. 2006b. Modelling the Lithospheric Magnetic Field Over France by Means of Revised Spherical Cap Harmonic Analysis. J. Geophys. Res.-Solid Earth, 111: B05102, 1-13.

VARENTSOV, IV. M. 1983. Modern Trends in the Solution of Forward and Inverse 3D Electromagnetic Induction Problems. Geophysical Surveys, 6: 55-78.

WEIDELT, P. 1971. The Electromagnetic Induction in two Thin Half-Sheets. Zeitschrift für Geophysik, 37: 649665.

WOLF, D. 1983a. Inductive Coupling Between Idealized Conductors and its Significance for the Geomagnetic Coast Effect. J. Geophys., 52: 22-33.

WOLF, D. 1983b. Singular Solutions to Maxwell's Equations and their Significance for Geomagnetic Induction. Geophys. J. R. Astr. Soc., 75: 279-283.

Received 10 February 2011

Accepted 21 November 2011 